Journal Club

Editor's Note: These short, critical reviews of recent papers in the Journal, written exclusively by graduate students or postdoctoral fellows, are intended to summarize the important findings of the paper and provide additional insight and commentary. For more information on the format and purpose of the Journal Club, please see http://www.jneurosci.org/misc/ifa_features.shtml.

\title{
Could an Allosteric Gating Model Explain the Role of TRPA1 in Cold Hypersensitivity?
}

\author{
Marcelo Salazar, ${ }^{1,2}$ Hans Moldenhauer, ${ }^{1,3}$ and David Baez-Nieto ${ }^{1,3}$ \\ ${ }^{1}$ Centro Interdisciplinario de Neurociencia Valparaíso, Facultad de Ciencias, Universidad de Valparaíso, Valparaiso 2349400, Chile, ${ }^{2} \mathrm{PhD}$ program in \\ Cellular and Molecular Biology, Facultad de Ciencias, Universidad Austral de Chile, Valdivia, Chile, and ${ }^{3}$ PhD program in Neuroscience, Facultad de \\ Ciencias, Universidad de Valparaiso, Valparaiso 2349400, Chile \\ Review of del Camino et al.
}

Mammalian transient receptor potential (TRP) channels consist of at least seven families of related proteins. A subset belong to the TRP channel superfamily, and they are responsible for temperature detection in mammals. These TRP channels (thermoTRPs) are expressed primarily in the dorsal root ganglion neurons in both somatosensory and nociceptive fibers. They respond to a wide range of temperatures from extreme cold $\left(<10^{\circ} \mathrm{C}\right)$ to extreme heat $\left(>42^{\circ} \mathrm{C}\right)$ (Latorre et al., 2009). TRPA1 is a cold-activated thermoTRP, and it is also activated by a broad spectrum of endogenous agonists and exogenous reactive irritants. Several of its activators are related to inflammatory processes. Although TRPA1 has been reported as an extreme cold receptor, its role in acute response to low temperatures is still controversial.

Using a heterologous expression, del Camino et al. (2010) confirmed findings by other groups (Sawada et al., 2007; Karashima et al., 2009) that TRPA1 channels and the cold-mediated activation of

Received Dec. 27, 2010; revised Feb. 1, 2011; accepted Feb. 4, 2011.

This work was supported by Fondo Nacional de Desarrollo Científico y Tecnológico Grant 1070049 and Comisión Nacional de Investigación Científica y Tecnológica fellowships. We thank to Drs. Ramon Latorre and Carlos Gonzalez for reading our manuscript, and for giving some suggestions that have been helpful to improve it. Centro Interdisciplinario de Neurociencia Valparaiso is a millenium institute.

Correspondence should be addressed to David Baez-Nieto, Centro Interdisciplinario de Neurociencias de Valparaiso, Gran Bretaña \#1111, Playa Ancha, Valparaiso 2349400, Chile. E-mail: david.baez@cinv.cl.

DOI:10.1523/JNEUROSCI.6775-10.2011

Copyright $\odot 2011$ the authors $\quad 0270-6474 / 11 / 315554-03 \$ 15.00 / 0$
TRPA1 current are $\mathrm{Ca}^{2+}$ independent. More importantly, del Camino et al. (2010) showed that TRPA1 elicited currents by allyl isothiocyanate (AITC) and other agonists were increased when the temperature was lowered from $25^{\circ} \mathrm{C}$ to $10^{\circ} \mathrm{C}$. These results conflict with those obtained by Karashima et al. (2009), who found that agonistinduced currents were reduced at lower temperatures. del Camino et al. (2010) explained that this discrepancy was due to a slower cooling system and the presence of extracellular calcium in the Karashima et al. (2009) experiment.

Figure 3 in del Camino et al. (2010) demonstrates that TRPA1 currents show a stronger increment when stimulated by AITC than by extreme cold. The increment is even greater when both stimuli are combined. Furthermore, when TRPA1 was stimulated by a highly saturated concentration of agonist [del Camino et al. (2010), their Fig. 3C] and the temperature lowered from $30^{\circ} \mathrm{C}$ to $10-20^{\circ} \mathrm{C}$, it produced comparable relative increases of currents. This result suggests that the equilibrium constant that governs the transition between the closed and open states is biased toward the open state by the presence of AITC.

The best model that can explain this bias is the allosteric kinetic model. This Journal Club paper aims to can clarify how TRPA1 might respond synergistically to different stimuli, such as temperature and agonists (Fig. 1A). Our model estab- lishes that different sensors (temperature and chemical) are located in different structural domains of the protein. Each structural domain is allosterically coupled to the channel gate, thus influencing the channel open probability $\left(P_{\mathrm{o}}\right)$.

The allosteric model for temperature and agonist concentration predicts that $P_{\mathrm{o}}$ as a function of temperature and agonist is given by the following equation:

$$
P_{\mathrm{o}}=\frac{1}{1+\frac{1+K+Q+K Q}{L(1+K C+Q F+K C Q F)}},
$$

where $L$ is the equilibrium constant between closed and open state, when all sensors are relaxed; $K$ and $Q$ are the constants that govern the temperature- and agonistdependent transitions; and $C$ and $F$ are the allosteric constants that couple the temperature and agonist sensors, respectively, with the channel gate.

Loss of sensitivity to other stimuli at high concentrations of agonist has been reported for other thermoTRPs, including TRPM8 and TRPV1 (Matta and Ahern, 2007). Our model supports these findings and predicts that in nonsaturated agonist concentration the relative increase of $P_{\mathrm{o}}$ in response to thermal stimulus should be greater (Fig. 1D) as reported by del Camino et al. (2010) (their Fig. 3C). Keeping in mind that a chemical stimulus causes more TRPA1 activity than cold, introducing a saturated 


\section{Kinetic model}

A

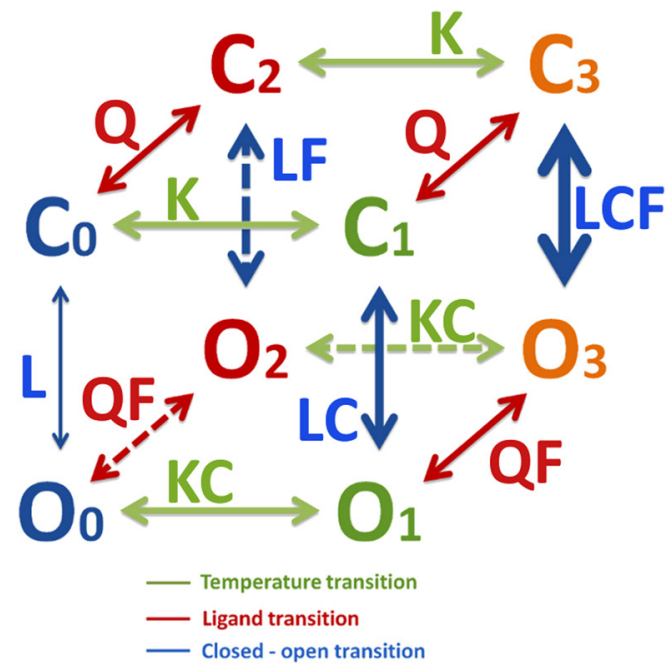

C

\section{Molecular model}

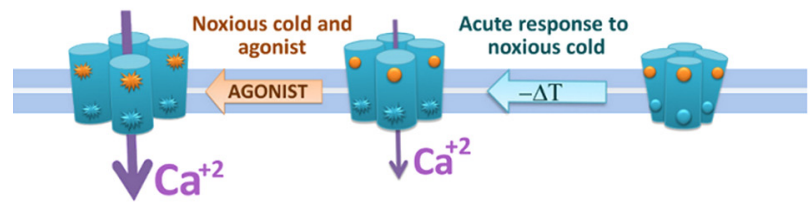

B

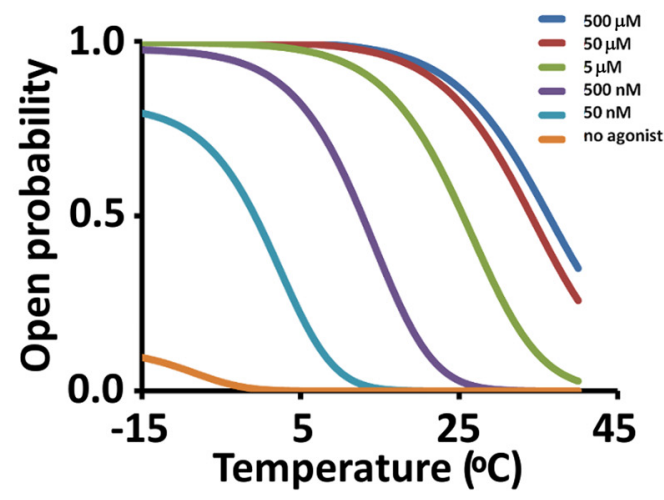

D

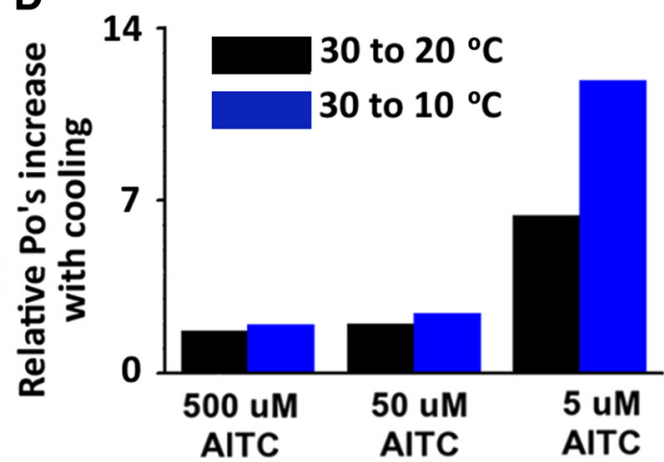

Figure 1. Kinetic and molecular models of TRPA1 channels. A, Allosteric model used to explain the TRPA1 activation by agonists and thermal stimulus. Note that when both stimuli are present, the equilibrium constant between closed and open state $(L)$ becomes $C F$ times larger, enhancing the channel activation. Nevertheless, the channel could visit the open states when all sensors are relaxed, typical behavior of an allosteric model. $\boldsymbol{B}$, Curves of probability versus temperature at different agonist concentrations using the kinetic model in $\boldsymbol{A}$. An increase of agonist concentration shifts to higher temperatures the $I_{1 / 2}$ value (obtained from Boltzmann equation fits), increasing the open probability of the channel, characteristic of the cold hypersensitivity process. At saturating agonist concentration, the channels become insensitive to cold, because they reach their maximum open probability, previously obtained by del Camino et al. (2010). Their work showed a minimum for TRPA1 channels activation in agonist's absence. This model fully supports the del Camino et al. (2010) findings. C, Activation mechanism of TRPA1 channel. The binding sites for agonists (orange balls) and the thermal sensors (blue balls) are located in different regions of the protein. They are coupling independently with the opening of the pore. When the extreme cold activates just the thermochannel sensors but their agonist binding sites still are empty, the $\mathrm{Ca}^{2+}$ influx is poor and thus the cold fiber detection is also poor and inadequate to generate action potentials. However, when both stimuli are present (the sensors are activated and the agonists have been binding with the channel), a massive entry of $\mathrm{Ca}^{2+}$ concentration produces a high level of membrane depolarization to result in TRPA1 channels with a dual role of detection in the nociceptive fibers involved with cold hypersensitivity. $D$, Relative increase of $P_{0}$ in response to temperature decrease from 30 to $10-20^{\circ} \mathrm{C}$ at different agonist concentrations. This graphic was generated by our proposed model. It is almost an exact reproduction of Figure 3C from del Camino et al. (2010). Here, del Camino et al. (2010) showed this relationship for the total relative current.

concentration of agonist will cause no response. The channel is now unresponsive to cold because it has already reached its maximum open probability.

We simulated the $P_{\mathrm{o}}$ of TRPAl as a function of temperature, in the presence of different AITC concentrations 0-500 $\mu \mathrm{M}$ (Fig. 1B). We used the allosteric model previously used for BK channels and modified for TRPM8 (cold and voltage) and TRPV1 (agonist) (Horrigan and Aldrich, 1999; Brauchi et al., 2004; Latorre et al., 2007; Matta and Ahern, 2007). The thermodynamic parameters for TRPA1 channel activation $[\Delta H=-28 \mathrm{~kJ} / \mathrm{mol}$ and $\Delta S=-107 \mathrm{~J} /(\mathrm{mol} \cdot \mathrm{K})]$ were obtained from Karashima et al. (2009). For the first approach, we used the values pre- viously published for TRPM8 channels for the allosteric constant that couples the temperature sensors and ligand sites with the pore $\left(L=4.4 \times 10^{-4}, C=1 \times 10^{4}\right.$, and $F=5 \times 10^{3}$, respectively) (Brauchi et al., 2004). For the dissociation constant $\left(K_{\mathrm{d}}\right)$, we used the $\mathrm{EC}_{50}$ value $(20 \mu \mathrm{M})$ of TRPA1 to AITC (Klionsky et al., 2007). Notice that the relative increase of currents [del Camino et al. (2010), their Fig. $3 C$ ] was fully reproduced by our proposed allosteric model for the $P_{\mathrm{o}}$ (Fig. $1 D$ ).

The increase in $P_{\mathrm{o}}$ magnitude is several times (fivefold) larger than the increase in the total macroscopic ionic current. This can easily be explained by the fact that the ionic current has a $Q_{10}$ of $\sim 1.5$. As shown in Equation 2, the macroscopic current, the unitary conductance $(\gamma)$, and the $P_{\mathrm{o}}$ are directly proportional to the current magnitude. If the temperature is reduced to $20^{\circ} \mathrm{C}$, the ionic current diminishes $66 \%$. The reduction is compensated by a 14 -fold increase in $P_{\mathrm{o}}$. Therefore, assuming that the number of channels remains constant during the experiment, there is a net increase in the magnitude of total currents despite the large reduction in the unitary conductance.

$$
I=N \cdot \gamma \cdot P_{\mathrm{o}} \cdot\left(V-V_{\text {rev }}\right)
$$

The main insight in the work of del Camino et al. (2010) was to clarify that synergistic response to different stimuli on TRPA1 channel. In this case the differ- 
ent stimuli were thermal and chemical, and they are not the algebraic sum of the responses to individual stimuli. Currents mediated by TRPA1 show a stronger increment in response to AITC than to those elicited by extreme cold alone. The response is even stronger when both stimuli are combined. This sensitization of TRPA1 mediated by agonists is present in heterologous as well as native expression systems. In DRG neurons primary culture, the channel is almost insensitive to extreme cold in the absence of any agonist [del Camino et al. (2010), their Fig. 3A, Fig. 4A]. We hypothesize that this channel functions as a dual detector that can trigger painful response when agonist and extreme cold are present.

del Camino et al. (2010) unveiled the in vivo role of TRPA1 by showing that the cold response in wild-type animals was dramatically increased by subcutaneous injection of an endogenous agonist produced during the inflammatory process (4-HNE) at nonlethal concentration. This response did not occur in trpa1 $1^{-/-}$transgenic mice. In the absence of agonist, transgenic mice have a normal response to extreme cold compared with wild type. However, TRPA1-null mice do not exhibit cold hypersensitivity after the agonist injection, indicating that TRPA1 does not account for all extreme cold perception. This mechanism of activation positions TRPA1 as a dual detector of inflammatory processes and extreme cold that triggers a painful re- sponse in the sensory terminal. This could underlie the cold hypersensitivity that occurs after tissue damage.

The data suggest a connection between the complex behavior described by del Camino et al. (2010) and the allosteric model we propose for thermoTRP activation. This model attempts to explain how the different ways to open the channels are integrated in the molecule. These changes are seen in the different amount of free energy involved in the channel gate opening. Thus the presence of both stimuli, thermal and chemical, activates both structurally independent sensors and produces a massive entry of $\mathrm{Ca}^{2+}$ (Fig. 1C).

del Camino et al. (2010) have made an important contribution in the field of thermoTRP channels by linking a complex mammal behavior to the functioning of a single protein. This allows us to connect TRPA1 allosteric activation and cold hypersensitivity, a very intricate and still elusive intracellular transduction mechanism. The allosteric model could be used to understand the different antagonists' concentration and their effectiveness to palliate pain. These results have major clinical implications as these channels are already targeted by several drugs designed to this exact purpose.

\section{References}

Brauchi S, Orio P, Latorre R (2004) Clues to understanding cold sensation: thermodynamics and electrophysiological analysis of the cold receptor TRPM8. Proc Natl Acad Sci U S A 101:15494-15499.

del Camino D, Murphy S, Heiry M, Barrett LB, Earley TJ, Cook CA, Petrus MJ, Zhao M, D’Amours M, Deering N, Brenner GJ, Costigan M, Hayward NJ, Chong JA, Fanger CM, Woolf CJ, Patapoutian A, Moran MM (2010) TRPA1 contributes to cold hypersensitivity. J Neurosci 30:15165-15174.

Horrigan FT, Aldrich RW (1999) Allosteric voltage gating of potassium channels II. Mslo channel gating charge movement in the absence of $\mathrm{Ca}(2+)$. J Gen Physiol 114:305-336.

Karashima Y, Talavera K, Everaerts W, Janssens A, Kwan KY, Vennekens R, Nilius B, Voets T (2009) TRPAl acts as a cold sensor in vitro and in vivo. Proc Natl Acad Sci U S A 106:1273-1278.

Klionsky L, Tamir R, Gao B, Wang W, Immke DC, Nishimura N, Gavva NR (2007) Speciesspecific pharmacology of trichloro(sulfanyl) ethyl benzamides as transient receptor potential ankyrin 1 (TRPA1) antagonists. Mol Pain 3:39.

Latorre R, Brauchi S, Orta G, Zaelzer C, Vargas G (2007) ThermoTRP channels as modular proteins with allosteric gating. Cell Calcium 42:427-438.

Latorre R, Zaelzer C, Brauchi S (2009) Structurefunctional intimacies of transient receptor potential channels. Q Rev Biophys 42:201-246.

Matta JA, Ahern GP (2007) Voltage is a partial activator of rat thermosensitive TRP channels. J Physiol 585:469-482.

Sawada Y, Hosokawa H, Hori A, Matsumura K, Kobayashi S (2007) Cold sensitivity of recombinant TRPA1 channels. Brain Res 1160: $39-46$. 